\title{
MODELAGEM DE MATERIAL COMBUSTÍVEL EM PLANTAÇÕES DE Pinus taeda NO NORTE DE SANTA CATARINA
}

\author{
Luiz Joaquim Bacelar de Souza ${ }^{1}$ \\ Ronaldo Viana Soares ${ }^{2}$ \\ Antonio Carlos Batista ${ }^{2}$
}

\begin{abstract}
RESUMO
Um inventário foi conduzido em Três Barras, Santa Catarina, para quantificar e modelar material combustível superficial vivo e morto em plantações de Pinus taeda. De forma sistemática foram estabelecidas 20 parcelas para cada povoamento de $3,5,7,9,11,13,15$, e 17 anos de idade, nas quais foram medidos a carga de combustível, o DAP, a altura e o diâmetro dominantes, a área basal e a espessura da liteira. Nove modelos foram ajustados através dos métodos Stepwise e "todas as regressões possíveis". O melhor modelo para estimar a carga de acículas foi $W a=2,356794 * S\left(\mathrm{R}^{2}=0,9563\right)$, ajustado com base na espessura $(S)$ da camada de acículas. Para a carga de combustível morto de até $2,5 \mathrm{~cm}$ de espessura $\mathrm{o}$ melhor modelo foi $W_{10}=1,889811+1,569117 * S+0,145218 * S^{2}\left(\mathrm{R}^{2}=0,8577\right)$. O modelo considerado mais adequado para determinação da carga total de combustível foi $W_{T}=3,35892 * S-1,87453\left(\mathrm{R}^{2}=0,7034\right)$. Três gráficos para estimativa indireta do combustível e um calibrador para estimar a carga de acículas são também apresentados. Palavras-chave: Combustível florestal, Modelagem de combustível, Plantação de Pinus, Proteção florestal.
\end{abstract}

FUEL MODELING IN Pinus taeda PLANTATIONS IN THE STATE OF SANTA CATARINA, BRAZIL

\begin{abstract}
A fuel inventory was conducted in Três Barras county, state of Santa Catarina, Brazil (26 $6^{\circ} 5^{\prime} \mathrm{S}$ latitude and $50^{\circ} 48^{\prime} \mathrm{W}$ longitude), in order to develop predicting models for fuel loading in loblolly pine (Pinus taeda) plantations. Sampling was done in 03, 05, 07, 09, 11, 13, 15, and 17-year-old stands, covering the whole rotation of the plantations. Twenty (20) plots of $1.0 \mathrm{~m}^{2}(1.0 \mathrm{x} 1.0 \mathrm{~m})$ were randomly located in each stand, totalizing 160 plots. The independent variables measured in the stands were age (in years), mean DBH $(\mathrm{cm})$, dominant DBH $(\mathrm{cm})$, dominant height of the trees $(\mathrm{m})$, basal area $\left(\mathrm{m}^{2}\right)$, and fuel bed depth $(\mathrm{cm})$. The dependent variables were live surface fuel, dead foliage (needles), dead woody fuel (separated by size classes), and total fuel load, all measured in ton.ha ${ }^{-1}$ (oven dry weight). Results showed good correlation between fuel bed depth and age and most of the dependent (fuel related) variables. Live surface fuel only presented significant correlation with mean DBH and basal area. However, live surface fuel was only significant in the 3-year-old plantation, and practically disappeared when the pines canopy intercepted most of the sun light, usually after the $5^{\text {th }}$ year. Models were developed to estimate the following variables: dead foliage $\left(\mathrm{W}_{\mathrm{a}}\right)$ dead fuel up to 10 -hour $(\varnothing \leq 2.5 \mathrm{~cm})$ timelag $\left(\mathrm{W}_{10}\right)$, and total fuel load $\left(\mathrm{W}_{\mathrm{T}}\right)$. Independent variables were chosen not only by the correlation coefficients, but also for the measuring facility, and based on these principles, fuel bed depth (S), age (I), dominant height (hdom), and dominant DBH (Ddom) were selected. The models were built through the Stepwise method, using the Statistic 5.0 software. Fuel bed depth was the variable that provided best fits for all predicting models, and the inclusion of other independent variables did not improve the models precision. The best model to estimate the needles load was $W a=2,356794 * S \quad\left(\mathrm{R}^{2}=0,9563\right)$; to estimate dead fine fuel load was $W_{10}=1,889811+1,569117 * S+0,145218 * S^{2} \quad\left(\mathrm{R}^{2}=0,8577\right) ;$ and to estimate the total fuel load was $W_{T}=3,35892 * S-1,87453\left(\mathrm{R}^{2}=0,7034\right)$. Total fuel load in the loblolly pine plantations in the studied site ranged from approximately 6.0 to 21.0 ton.ha ${ }^{-1}$.
\end{abstract}

Keywords: Forest fuel, Fuel modeling, Pine plantations, Forest protection.

\footnotetext{
${ }^{1}$ Professor do Curso de Engenharia Florestal da Universidade Federal do Amazonas

${ }^{2}$ Professor do Departamento de Ciências Florestais da Universidade Federal do Paraná 


\section{INTRODUÇÃO}

Em vista da grande ameaça que os incêndios representam para as florestas e povoamentos florestais do planeta, aumenta a cada dia a preocupação com a implantação de eficientes sistemas integrados de proteção contra incêndios florestais. Esses sistemas, operando com recursos de informática, possibilitam automaticamente a detecção e localização do fogo, estimando sua intensidade e indicando rotas de acesso, além dos recursos necessários para combatê-lo. A qualidade das informações relacionadas ao comportamento do fogo é de suma importância para garantir a eficiência de tais sistemas, principalmente no que tange às operações de combate, cujo planejamento depende fundamentalmente dos dados relativos à quantidade de material combustível. Esta informação, é também indispensável para a prescrição de queimas controladas em áreas florestais, seja para a redução do risco de ocorrência de incêndios ou para se atingir outros objetivos previstos no plano de manejo florestal.

Embora um inventário seja a forma mais precisa de quantificação do combustível florestal, o tempo despendido na sua realização é muito longo, o que inviabiliza sua utilização nos casos em que o incêndio já iniciou e se pretende estimar a intensidade do fogo. Nestas situações o mais indicado é recorrer aos modelos de combustível para estimar, a qualquer momento, através de variáveis de fácil obtenção, a carga de material combustível existente numa floresta.

No Brasil, modelos como esses praticamente inexistem. Houve somente uma tentativa de desenvolvimento de equações de predição da quantidade de combustível para Pinus caribaea e $P$. oocarpa no sudoeste de Minas Gerais, em povoamentos jovens, com até sete anos e meio de idade (Soares, 1979), quando o ideal seria poder dispor de modelos que cubram todo o período de rotação da floresta.

A escolha do Pinus taeda para se iniciar a elaboração de modelos de material combustível deve-se ao fato dela ser atualmente a espécie mais plantada no sul do Brasil, apresentando grande importância para a economia florestal da região, além da facilidade de se encontrar todas as idades do período de rotação em um mesmo distrito florestal.

Este trabalho teve como objetivo principal o desenvolvimento de modelos matemáticos para se estimar a quantidade de material combustível em plantações de Pinus taeda de diferentes idades e não submetidas a regime de desbastes, através de variáveis de fácil obtenção, tais como idade, altura, diâmetro e espessura da camada de acículas.

\section{METODOLOGIA}

\section{Localização da área de estudo}

Este trabalho foi desenvolvido em um distrito florestal de propriedade da empresa RIGESA, Celulose, Papel e Embalagens Ltda., localizado em Três Barras, SC, em povoamentos de Pinus taeda de diferentes idades, plantados em espaçamento inicial de $2,5 \times 2,5 \mathrm{~m}$. A região abrangida pela pesquisa situa-se em média a $26^{\circ} 15^{\prime}$ de latitude sul e $50^{\circ} 48^{\prime}$ de longitude oeste e a $775 \mathrm{~m}$ de altitude. Os talhões amostrados apresentavam geralmente topografia ligeiramente plana a levemente ondulada e localizavam-se em regiões administrativas da RIGESA denominadas de Bugre, Paul, Caraguatá, Paredão, São João da Barra, Experimental, Duas Barras e Divisa.

\section{Amostragem}

O inventário básico foi feito através da medição e pesagem do material combustível vivo e morto localizado entre a camada $\mathrm{H}$ do horizonte orgânico e $1,8 \mathrm{~m}$ de altura, em parcelas de 1,0 $\mathrm{m} 2$, distribuídas uniformemente sobre todos os talhões estudados, nos plantios de idades impares $(3,5,7,9,11,13,15$ e 17 anos). O formato quadrado das parcelas (Figura 1) foi adotado com base nas pesquisas de Soares (1979); Batista (1984,1995); Schneider e Bell (1985) e de Ribeiro (1997). A amostragem foi realizada entre os meses de julho e outubro de 1997. 


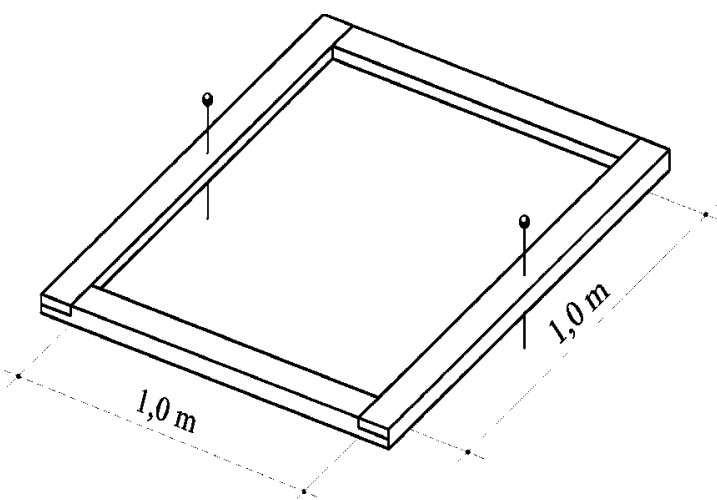

Figura 1: Gabarito utilizado para amostragem do material combustível

Figure 1: Gauge used to sample fuel load

De acordo com as experiências de Brown (1971) ; Soares (1979); Carlton e Pickford (1982) foram coletadas 20 amostras para cada idade de plantio, perfazendo um total de 160. O número final de amostras foi determinado com base na variância das mesmas, através do método seqüencial de Stein (Steel e Torrie, 1980). A população foi considerada como infinita e o limite de erro tolerado para a determinação do número de amostras foi pré-estabelecido em $10 \%$. O nível de probabilidade adotado foi de $90 \%$, valor compatível com trabalhos da área biológica.

\section{Obtenção das variáveis explicativas}

Após a fixação do gabarito para coleta de material combustível, mediram-se as circunferências à altura do peito (CAP) das árvores que cobriam uma área correspondente ao espaço inicialmente ocupado pelo plantio inicial de 36 mudas ( $6 \times 6$ plantas) ao redor da parcela. A partir destes dados foram determinados o DAP médio das árvores e a área basal $(\mathrm{G})$ do talhão. Além destes parâmetros, em cada ponto de amostragem, foram medidas a profundidade média da camada de acículas (S) e a altura média das árvores dominantes (hdom). A profundidade da camada de acículas foi medida com auxílio de uma régua graduada com precisão de $0,1 \mathrm{~cm}$, nos quatro cantos e no centro do gabarito, obtendo-se daí o valor médio desta variável para cada parcela.

\section{Determinação da carga de material combustível}

Antes da separação e pesagem do combustível morto delimitado pelo gabarito a vegetação viva foi cortada rente ao piso do povoamento, pesada e homogeneizada para a retirada de uma sub-amostra que, depois de pesada, foi levada para secagem em laboratório. Nesta operação foram usados um facão e um serrote de poda para facilitar a remoção e posterior classificação de todo o material contido na área de um metro quadrado. O material lenhoso (fragmentos de ramos, cones, cascas, restos de exploração e estruturas da vegetação baixa morta) foi classificado em quatro diferentes padrões de diâmetro $(\phi 1 \leq 7 \mathrm{~mm} ; \phi 2$ de 7,1 a $25 \mathrm{~mm} ; \phi 3$ de 25,1 a $75 \mathrm{~mm}$ e $\phi 4>75 \mathrm{~mm}$ ), com auxílio de um calibrador de fibra (Figura 2),

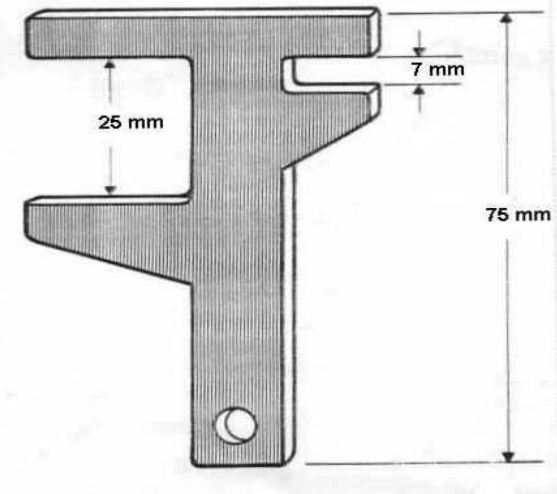


Figura 2: Calibrador utilizado para separação das classes de material combustível (BROWN 1974)

Figure 2: Gauge used to separate dead fuel size classes

Um gabarito menor (Figura 3), com as mesmas dimensões $(20 \times 20 \mathrm{~cm})$ usadas por Hingston e Galbraith (1989) foi usado para facilitar a retirada das sub-amostras de acículas da manta delimitada pelo gabarito maior.

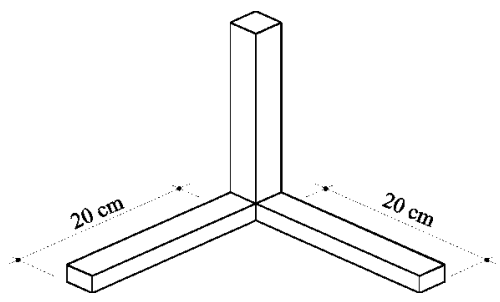

Figura 3: Gabarito utilizado na coleta de acículas Figure 3: Gauge used to sample dead needles

Depois da identificação e separação dos materiais foi feita a pesagem das acículas e das diferentes classes de material lenhoso. Posteriormente, foram retiradas as respectivas sub-amostras, que depois de pesadas foram levadas ao laboratório para secagem a uma temperatura de $\pm 80^{\circ} \mathrm{C}$ até atingir peso constante.

Devido às dimensões de algumas classes $(>25 \mathrm{~mm})$, todas as sub-amostras sofreram pré-secagem contínua numa câmara de abertura de cones por um período nunca inferior a cinco dias, a uma temperatura de aproximadamente $28^{\circ} \mathrm{C}$. A pesagem do material, úmido e seco, foi feita em uma balança com capacidade de 2610 g e precisão de $0,1 \mathrm{~g}$, no campo e no laboratório, respectivamente.

A quantidade, em peso de matéria seca (W), para os diferentes tipos e classes de material combustível foi calculada da seguinte forma:

$$
W=\left(\frac{\text { Peso da sub - amostra SECA } \times \text { Peso da amostra ÚMIDA }}{\text { Peso da sub-amostra ÚMIDA }}\right) \times 10 \quad(\mathrm{Mg} / \mathrm{ha})
$$

Foram estabelecidas as seguintes classes de material combustível:

$\mathrm{W}_{\mathrm{a}} \rightarrow$ acículas das camadas L e F da serapilheira;

$\mathrm{W}_{1}\left[\mathrm{~W}_{\mathrm{a}}+\mathrm{W} \phi_{1}\right] \rightarrow$ acículas + combustíveis da classe $\phi_{1}$;

$\mathrm{W}_{10}\left[\mathrm{~W}_{1}+\mathrm{W}_{2}\right] \rightarrow \mathrm{W}_{1}+$ combustíveis da classe $\phi_{2}$;

$\mathrm{W}_{100}\left[\mathrm{~W}_{10}+\mathrm{W} \phi_{3}\right] \rightarrow \mathrm{W}_{10}+$ combustíveis da classe $\phi_{3}$;

$\mathrm{W}_{\mathrm{k}}\left[\mathrm{W}_{100}+\mathrm{W} \phi_{4}\right] \rightarrow \mathrm{W}_{100}+$ combustíveis da classe $\phi_{4}$;

$\mathrm{W}_{\mathrm{v}} \rightarrow$ combustível superficial vivo

$\mathrm{W}_{\mathrm{T}} \rightarrow$ combustível total $\left[\mathrm{W}_{\mathrm{v}}+\mathrm{W}_{\mathrm{k}}\right]$

\section{Modelagem}

Antes da montagem da matriz de correlação das variáveis de potencial interesse na modelagem, os dados relacionados ao material combustível foram submetidos a uma verificação da homogeneidade das variâncias através da aplicação do teste de Bartlett. Na expectativa de obtenção de melhores ajustes algumas destas variáveis sofreram transformações, dependendo de suas características e da fase de desenvolvimento das análises.
O grau de relacionamento das variáveis explicativas ou independentes com as quantidades de material combustível foi obtido através da montagem de uma matriz de correlação envolvendo todas as variáveis, de modo a permitir uma seleção daquelas com os melhores coeficientes, conforme escala adotada por Schmidt (1977), ou seja, até 0,30 baixa; de 0,31 a 0,60 - média; de 0,61 a 0,80 forte e acima de 0,80 - muito forte. Com base nos resultados da matriz foram selecionadas apenas as variáveis DAP médio, idade e 
espessura média da camada de acículas para composição dos modelos.

Assim como as variáveis explicativas, as variáveis de resposta ou dependentes foram previamente definidas a partir de critérios de ordem prática, quais sejam: baixa propensão a erro, relação com o sítio e facilidade de obtenção; no entanto, o principal critério foi o grau de importância das mesmas no contexto dos incêndios florestais. As equações para estimar a quantidade de material combustível foram ajustadas a partir destas variáveis e das variáveis explicativas também selecionadas.

Dois métodos foram combinados para ajustar os modelos, Stepwise e "todas as regressões possíveis", permitindo o estudo de um expressivo número de equações e a préseleção das melhores. Diversos foram os modelos testados (linear simples e múltiplo, polinomial, exponencial, logaritmo e potencial). Os ajustes das equações foram avaliados através do coeficiente de determinação ajustado (R2), do erro padrão da estimativa como percentagem da média estimada (Syx\%), da razão de variância $F$ e pela análise de resíduos. No ajuste das equações não lineares foi utilizado preliminarmente o método Quasi-Newton, com posterior determinação do R2 ajustado e erro padrão da estimativa pelo método dos Mínimos Quadrados. Os modelos não lineares ajustados foram obtidos principalmente a partir de inferências e adaptações a modelos de mesma natureza, sem que no entanto fossem preteridas as análises exploratórias feitas sob bases diagramáticas.

Além dos critérios estatísticos, os modelos foram escolhidos com base nas características das variáveis explicativas e de resposta e na sua composição. As características consideradas foram: facilidade de obtenção da variável, precisão que a medição da variável permite, grau de relação de dependência de fatores do sítio e intercorrelação entre as mesmas, quando se tratava de duas ou mais integrando o mesmo modelo. Além do mais, o nível de multiplicidade de variáveis ou complexidade do modelo também foi considerado como critério de seleção, favorecendo os modelos mais simples. Em relação à variável resposta foram considerados o potencial de abrangência do modelo e sua posição hierárquica no contexto dos níveis de periculosidade, o qual foi determinado com base no timelag, parâmetro estritamente dependente do diâmetro do material lenhoso que é a dimensão controladora da velocidade do fluxo de umidade.

Após a escolha dos melhores modelos para cada variável, foram construídos gráficos para facilitar a operacionalização do modelo. Com base no melhor modelo ajustado foi desenvolvido um calibrador de carga (régua graduada) para estimar a carga de acículas nas plantações daquele distrito florestal.

\section{RESULTADOS E DISCUSSÃO}

\section{Carga de combustível}

A Tabela 1, elaborada a partir do inventário, mostra a variação da quantidade de material combustível, nas diferentes classes, em função da idade do povoamento. Os combustíveis mortos, representados pelas acículas, miscelânea e material lenhoso, apresentaram maior contribuição na carga total de combustível superficial a partir do fechamento do dossel. 
Tabela 1: Distribuição das cargas de combustíveis superficiais das plantações de Pinus taeda de diferentes idades por classe padrão

Table 1: $\quad$ Standard classes distribution of surface fuel loads in loblolly pine plantations of different ages

\begin{tabular}{lcccccccc}
\hline CLASSE & \multicolumn{7}{c}{ CARGA DE COMBUSTÍVEL SECO (Mg/ha) POR IDADE (anos) } \\
\cline { 2 - 9 } PADRÃO & 3 & 5 & 7 & 9 & 11 & 13 & 15 & 17 \\
\hline $\mathrm{W}_{\mathrm{a}}$ & 0,00 & 5,53 & 7,33 & 8,62 & 13,21 & 10,89 & 16,69 & 18,01 \\
$\mathrm{~W}_{\phi 1}$ & 0,27 & 0,20 & 0,32 & 0,57 & 0,56 & 0,77 & 1,08 & 1,19 \\
$\mathrm{~W}_{\phi 2}$ & 1,63 & 0,20 & 0,57 & 1,24 & 0,85 & 1,10 & 2,24 & 2,05 \\
$\mathrm{~W}_{\phi 3}$ & 0,76 & 0,01 & 0,00 & 0,43 & 0,16 & 0,54 & 0,57 & 0,44 \\
$\mathrm{~W}_{\phi 4}$ & 2,36 & 0,00 & 0,00 & 0,54 & 0,00 & 0,00 & 0,08 & 0,14 \\
$\mathrm{~W}_{\mathrm{v}}$ & 8,95 & 0,13 & 0,01 & 0,00 & 0,00 & 0,00 & 0,00 & 0,04 \\
$\mathrm{~W}_{\mathrm{T}}$ & 13,97 & 6,07 & 8,10 & 11,39 & 13,70 & 13,39 & 20,66 & 21,10 \\
\hline
\end{tabular}

O combustível representado pelo material morto contido na serrapilheira, com exceção do povoamento de 3 anos, apresentou quantidades crescentes a partir das idades mais jovens, demonstrando que até a idade de 17 anos a acumulação deste material ainda não havia estacionado, ou seja, o processo de deposição continuava sobrepondo-se ao processo de decomposição.

Uma quantidade expressiva de vegetação viva de pequeno porte foi registrada nos povoamentos de 3 anos de idade. Daí em diante a proporção entre combustível vivo e morto se inverteu, caindo de maneira expressiva na idade subseqüente. A partir dos 9 anos a quantidade de combustível vivo atingiu o valor zero, mantendo-se praticamente neste nível até o final da rotação. A maior quantidade de luz incidente sobre o piso dos talhões nas idades mais jovens certamente favoreceu $o$ desenvolvimento da vegetação herbácea, arbustiva e até mesmo de alguns indivíduos oriundos de regeneração natural de plantações mais velhas existentes nas proximidades. Segundo BRENDER et al. (1976), em povoamentos de idades mais jovens, a vegetação invasora constitui a maior fração de combustível, enquanto nas idades mais avançadas, o material morto do piso florestal chega a contribuir com até $97 \%$ da referida carga, o que está de acordo com os resultados obtidos na presente pesquisa.

Da carga total de combustível morto, a maior proporção foi devida à massa de acículas, com pequena contribuição dos materiais lenhosos. Excetuando-se as duas primeiras idades estudadas, os combustíveis com diâmetros acima de $25 \mathrm{~mm}$ tiveram muito pequena contribuição na quantidade total. Como conseqüência, pode-se dizer que, em relação aos povoamentos estudados, as acículas se constituíram na única classe capaz de representar com boa aproximação a carga total de combustíveis.

\section{Seleção das variáveis}

Partindo de um total de 18 variáveis, sendo 6 independentes e 12 dependentes e adotando os princípios mencionados por Draper \& Smith (1966), Vanclay (1994) e Werkema \& Aguiar (1996), elas foram submetidas a sucessivos processos de eliminação até serem reduzidas a menos da metade. Por isto, das doze variáveis dependentes preliminarmente eleitas, apenas três continuaram a fazer parte da análise.

De acordo com a Tabela 2, apenas os combustíveis com diâmetro acima de $75 \mathrm{~mm}$ e os combustíveis vivos apresentaram correlação negativa com as variáveis independentes, o que permite inferir que os combustíveis destas classes tendem a diminuir quando a idade da plantação ou os valores das demais variáveis aumentam. No entanto, como as maiores quantidades de combustíveis grossos estão concentradas em idades baixas (Tabela 1), estas cargas podem ser interpretadas como o resultado da competição por luz associada a grandes quantidades de resíduos da exploração passada. Quanto ao combustível vivo, a tendência de declínio pode ser atribuída às mudanças de luminosidade proporcionadas pelo fechamento do dossel, principalmente nas idades mais jovens. 
Tabela 2: Coeficientes de correlação entre as variáveis medidas e as classes de material combustível nos povoamentos de Pinus taeda

Table 2: Correlation coefficients between measured variables and fuel classes in loblolly pine plantations

\begin{tabular}{lcccccc}
\hline \multirow{2}{*}{ VARIÁVEIS } & \multicolumn{5}{c}{ VARIÁVEIS INDEPENDENTES } \\
\cline { 2 - 7 } DEPENDENTES & Idade & $\mathrm{G}$ & $\mathrm{S}$ & $\mathrm{DAP}$ & hdom & Ddom \\
\hline $\mathrm{W}_{\mathrm{a}}$ & 0,787 & 0,704 & 0,883 & 0,752 & 0,735 & 0,756 \\
$\mathrm{~W}_{\phi 1}$ & 0,676 & 0,522 & 0,600 & 0,542 & 0,657 & 0,569 \\
$\mathrm{~W}_{\phi 2}$ & 0,336 & 0,187 & 0,267 & 0,175 & 0,302 & 0,212 \\
$\mathrm{~W}_{\phi 3}$ & 0,147 & 0,023 & 0,037 & 0,011 & 0,123 & 0,030 \\
$\mathrm{~W}_{\phi 4}$ & $-0,273$ & $-0,345$ & $-0,339$ & $-0,374$ & $-0,279$ & $-0,356$ \\
$\mathrm{~W}_{01}$ & 0,807 & 0,716 & 0,894 & 0,763 & 0,756 & 0,770 \\
$\mathrm{~W}_{10}$ & 0,812 & 0,702 & 0,883 & 0,745 & 0,759 & 0,757 \\
$\mathrm{~W}_{100}$ & 0,812 & 0,690 & 0,869 & 0,731 & 0,757 & 0,745 \\
$\mathrm{~W}_{\mathrm{k}}$ & 0,773 & 0,632 & 0,817 & 0,668 & 0,715 & 0,685 \\
$\mathrm{~W}_{\mathrm{v}}$ & $-0,480$ & $-0,639$ & $-0,568$ & $-0,686$ & $-0,492$ & $-0,627$ \\
$\mathrm{~W}_{\mathrm{T}}$ & 0,568 & 0,334 & 0,568 & 0,347 & 0,500 & 0,398 \\
\hline
\end{tabular}

Os coeficientes de correlação fornecidos pela Tabela 2 permitiram a constatação das fracas associações entre as classes definidas por faixas de dimensões complementares $\left(\mathrm{W}_{\phi \mathrm{n}}\right)$ e as 6 variáveis independentes. Por esta razão as referidas classes de combustível foram preteridas em favor das classes cumulativas definidas com base no timelag.

A forte correlação entre as variáveis independentes e as classes de combustível não foi o único critério usado para selecionar as variáveis de resposta ou dependentes. Algumas características relacionadas às classes cumulativas $\left(\mathrm{W}_{\mathrm{n}}\right)$ também foram levadas em consideração, principalmente a relação entre flamabilidade e espessura do material combustível. A classe " $\mathrm{W}_{10}$ " foi a que melhor representou o balanço entre o grau de associação com as variáveis independentes e participação na carga total " $\mathrm{W}_{\mathrm{T}}$ " de combustíveis (Tabelas 1 e 2). A opção por esta representante das classes cumulativas também levou em conta os argumentos apresentados por Sackett (1980), Agee (1983), Chandler et al. (1983), Pyne (1984), Brown e Bevins (1986), McCaw et al.(1996) e Schimmel e Granström (1997), os quais fazem referências à importância dos combustíveis de diâmetros menores do que $25 \mathrm{~mm}$, que geralmente são consumidos pela maioria dos incêndios e queimas controladas.

Além da variável de resposta " $\mathrm{W}_{10}$ " foram escolhidas ainda as variáveis referentes à carga de acículas $\left(\mathrm{W}_{\mathrm{a}}\right)$ e à carga superficial total $\left(\mathrm{W}_{\mathrm{T}}\right)$, devido a importância destas informações no que diz respeito à avaliação do potencial de risco de incêndio.

Com relação às variáveis independentes, a profundidade da manta de acículas e a idade do povoamento apresentaram as mais fortes correlações com as variáveis dependentes modeladas $\left(\mathrm{W}_{\mathrm{a}}, \mathrm{W}_{10} \mathrm{e}\right.$ $\mathrm{W}_{\mathrm{T}}$ ). O DAP apresentou fortes correlações com combustível vivo (não modelado) e com a $\mathrm{W}_{\mathrm{a}}$; a altura dominante, com a $\mathrm{W}_{10}$ e a $\mathrm{W}_{\mathrm{T}}$; e o diâmetro dominante com a $\mathrm{W}_{\mathrm{a}}$ e a $\mathrm{W}_{10}$. Com base nesses resultados, essas cinco variáveis independentes foram usadas na modelagem das variáveis de resposta.

Modelos ajustados

A Tabela 3 apresenta três equações que estimam a carga de acículas com base na espessura da camada de acículas e na idade do povoamento visto que a inclusão de outras variáveis independentes não melhorou o ajuste dos modelos. O modelo $\mathrm{n}^{\circ} 01$, baseado apenas na espessura da camada de acículas, além de ser mais simples para se calcular, apresentou maior $\mathrm{R}^{2}$ do que as demais e por estes motivos foi selecionado como o mais indicado para a estimativa da carga de acículas em povoamentos de Pinus taeda. No entanto, o modelo $\mathrm{n}^{\mathrm{o}} 02$ não deve ser totalmente descartado pois se baseia apenas na idade do povoamento, variável fácil de se obter e que não necessita de medições de campo. 
Tabela 3. Modelos ajustados para estimar a carga de acículas da serapilheira de Pinus taeda

Table 3. Adjusted models to estimate dead needles load in loblolly pine plantations.

\begin{tabular}{lrrrrr}
\hline \multirow{2}{*}{ MODELOS } & $\mathrm{N}^{\mathrm{o}}$ & \multicolumn{2}{c}{ ESTATÍSTICAS } & \\
\cline { 4 - 6 }$W_{a}=2,356794 * S$ & {$[01]$} & 0,9563 & 2,264 & $* *$ \\
\hline$W_{a}=\left(7,19864-0,000112 * I^{3}-\frac{12,016474}{\sqrt{I}}\right)^{2}$ & {$[02]$} & 0,7172 & 3,089 & $* *$ \\
\hline$W_{a}=e^{0,706486+2,177233^{2} l_{n} \sqrt{S}}$ & {$[03]$} & 0,8649 & 2,128 & $* *$ \\
\hline
\end{tabular}

** Significativo ao nível de $95 \%$

A ausência de um intercepto no modelo $n^{\circ}$ 01 deve-se ao fato do mesmo não ter sido significativo ao nível de $95 \%$ de probabilidade. Sua remoção aumentou sensivelmente $o R^{2}$ e reduziu o $\mathrm{S}_{\mathrm{yx}}$, favorecendo a seleção deste modelo entre os três apresentados. Os diagramas de distribuição dos resíduos reforçaram as considerações em relação ao citado modelo.

Trabalhando com P. taeda no norte do Paraná, Batista (1995) obteve equações exponenciais, também baseadas na espessura da manta de acículas, com valores de R2 variando de 0,81 a 0,86 e de Syx variando de 2,2304 a 2,2680, bem próximos aos observados na presente pesquisa, o que demonstra a viabilidade do uso dessa variável independente na determinação da carga de acículas. Outros autores, McRae et al. (1979) e Burrows et al. (1989) também obtiveram bons resultados usando a mesma variável independente para estimar a carga de acículas em povoamentos de Pinus sp.

A título de ilustração poderia se dizer que a espessura da camada de acículas está para a sua massa assim como o DAP de uma árvore está para o seu volume ou peso, ou seja, são variáveis diretamente envolvidas com o objeto fim e por isso resultam em relações mais precisas em análises de regressão. No entanto, a espessura da camada de acículas, ao contrário da árvore, sofre a ação simultânea de dois processos, que são a deposição e a decomposição. Estes processos, assim como a produção florestal, dependem, além de suas peculiaridades, dos fatores do sítio e suas correspondentes oscilações. A diferença reside no fato de que as árvores não sofrem oscilações positivas e negativas (crescem mas não decrescem), ou melhor, árvores vivas não sofrem redução dimensional cuja progressão seja mensurável. Entretanto, no caso da camada de acículas, o resultado da ação simultânea dos processos que regem a carga de matéria morta pode, em um determinado momento, atingir níveis decrescentes quando a decomposição supera a deposição. Assim sendo, mesmo baseando-se em variáveis de ligação direta como a espessura da camada, o processo de modelagem está muito mais sujeito a erros, provocados pela referida dinâmica, do que os modelos de produção florestal. Por essa razão, as equações para estimar a carga de acículas ou mesmo de outras classes de combustível morto, precisam ser estudadas sob os mais variados prismas, considerando sua sensibilidade às oscilações ambientais como secas, enchentes e outras contribuições de caráter climatológico.

A Tabela 4 apresenta os modelos selecionados para estimar a carga de combustíveis com timelag de até 10 horas, que inclui as acículas e o material lenhoso de $\varnothing \leq$ $2,5 \mathrm{~cm}$, considerado o mais importante para a ocorrência e propagação dos incêndios. 
Tabela 4: Modelos ajustados para estimar carga de combustíveis com timelag de até 10 horas nas plantações de Pinus. taeda.

Table 4. Adjusted models to estimate fuel load up to 10 hour timelag in loblolly pine plantations

\begin{tabular}{|c|c|c|c|c|}
\hline \multirow{2}{*}{ MODELOS } & \multirow{2}{*}{$\mathrm{N}^{\mathrm{o}}$} & \multicolumn{3}{|c|}{ ESTATÍSTICAS } \\
\hline & & $\mathrm{R}^{2}$ & $\mathrm{~S}_{\mathrm{yx}}$ & $\mathrm{F}_{0,95}$ \\
\hline$W_{10}=1,889811+1,569117 * S+0,145218 * S^{2}$ & {$[04]$} & 0,8577 & 2,418 & $* *$ \\
\hline$W_{10}=\left(1,690201 * l_{n} I-0,495039\right)^{2}$ & {$[05]$} & 0,7025 & 3,507 & $* *$ \\
\hline$W_{10}=2,415374+\frac{S^{1,595280}}{e^{-0.033069^{*} S}}$ & [06 ] & 0,8514 & 2,463 & $* *$ \\
\hline
\end{tabular}

** Significativo ao nível de 95\%

Pelas estatísticas correspondentes e respectivas distribuições de resíduos, os modelos [04] e [06] poderiam ser considerados equivalentes, ambos com uma variável explicativa e 3 coeficientes, porém o primeiro apresenta coeficiente de determinação um pouco mais alto e é mais simples para se calcular. Quanto ao modelo [05], que depende apenas da idade, pode ser importante quando não houver disponibilidade da espessura da camada de acículas.

A Tabela 5 apresenta os modelos mais indicados para a estimativa da carga total de combustíveis em povoamentos de Pinus taeda na região estudada.

Tabela 5: Modelos ajustados para estimar a carga total de combustível superficial em plantações de Pinus taeda.

Table: Adjusted models to estimate surface total fuel load in loblolly pine plantations

\begin{tabular}{|c|c|c|c|c|}
\hline \multirow{2}{*}{ MODELOS } & \multirow{2}{*}{$\mathrm{N}^{\mathrm{o}}$} & \multicolumn{3}{|c|}{ ESTATISTICAS } \\
\hline & & $\mathrm{R}^{2}$ & $\mathrm{~S}_{\mathrm{yx}}$ & $\mathrm{F}_{0,95}$ \\
\hline$W_{t}=3,35892 * S-1,87453$ & {$[07]$} & 0,7034 & 3,770 & $* *$ \\
\hline $\log W_{t}=0,32849+\frac{2,34190}{I S^{2}}+0,18223 \sqrt{I S}-0,02236 * I^{2} \sqrt{S}$ & {$[08]$} & 0,6819 & 3,368 & $* *$ \\
\hline$W_{t}=6,467589+\frac{S^{-0,173951}}{e^{-0,415063 . S}}$ & [09] & 0,5858 & 3,818 & $* *$ \\
\hline
\end{tabular}

De acordo com as estatísticas apresentadas na Tabela 5, a precisão da equação não linear [09] foi bem inferior do que as outras [07] e [08], que apresentaram ajustes semelhantes, apesar de suas características estruturais serem distintas: uma com uma variável e um coeficiente, outra com duas variáveis em três combinações e quatro coeficientes. A distribuição dos resíduos confirmou a superioridade do modelo [07] sobre os demais.

A diminuição da precisão de ajuste dos modelos que estimam a carga total de combustíveis em relação aos que estimam as cargas de acículas e de combustíveis finos $(\varnothing$ $\leq 2,5 \mathrm{~cm}$ ), observada através dos coeficientes de determinação, deve-se à grande variabilidade observada nas cargas de combustíveis vivos e de dimensões maiores $(\varnothing$ $>2,5 \mathrm{~cm}$ ). Provavelmente pelo mesmo motivo, não se obteve bom ajuste para um modelo baseado apenas na idade do povoamento.

\section{Aplicações dos modelos}

Os modelos selecionados podem servir de base para a elaboração de tabelas ou instrumentos que possam ser utilizados na avaliação de cargas de material combustível, como mostra a figura 4. 


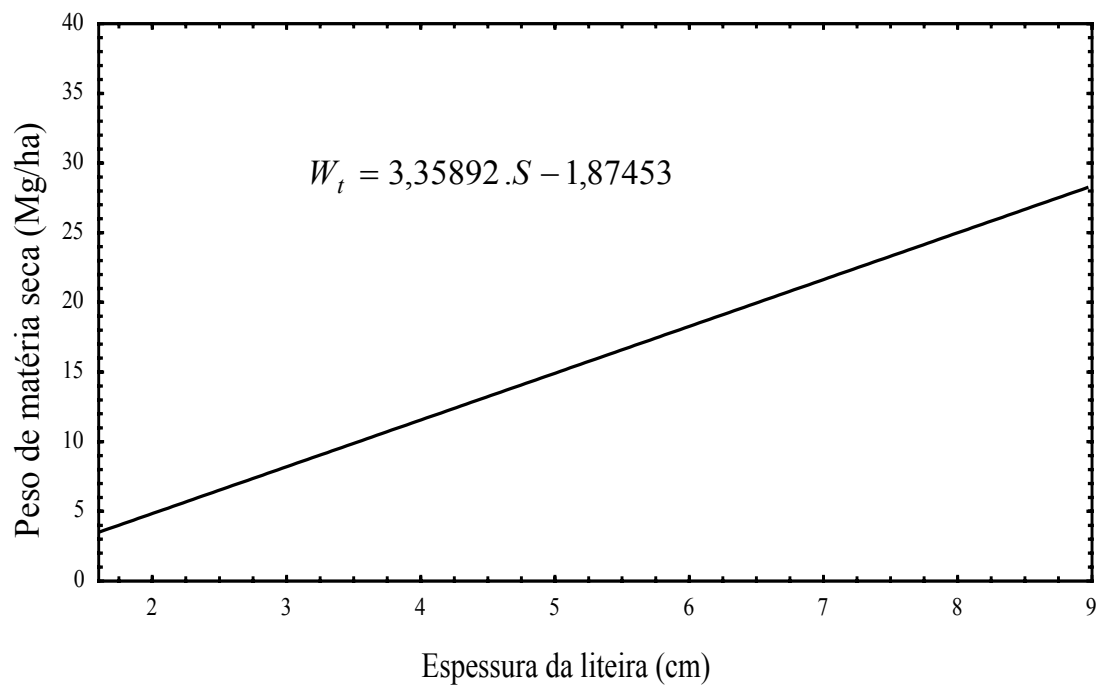

Figura 4: Estimativa da carga de combustível superficial total a partir da espessura da camada orgânica depositada sobre o solo

Figure 4. Total surface fuel load estimation through the organic layer thickness

Os resultados da pesquisa permitiram também a construção de uma régua plástica graduada (Figura 5) capaz de estimar diretamente, em $\mathrm{Mg} / \mathrm{ha}$, a carga de acículas dos povoamentos de Pinus taeda da região estudada, através da equação [01]. A extremidade pontiaguda facilita sua utilização, permitindo uma penetração suave no solo; quando uma maior resistência for percebida, significa que a região basal ou nível zero da régua se encontra no nível ideal para a realização da leitura da carga de acículas. Denominado nesta proposta de "Calibrador de carga", este instrumento de fácil manuseio pode oferecer maior rapidez do que a maioria dos métodos, diretos e indiretos, na estimativa do peso das acículas.

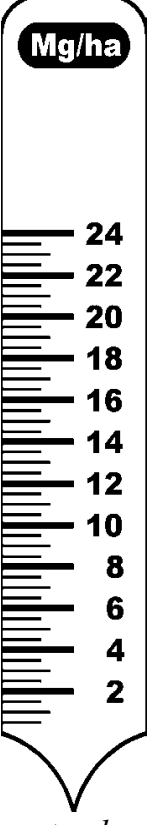

Figura 7. Calibrador de carga para acículas de Pinus taeda

Figure 7. Gauge to estimate dead needles load in loblolly pine plantations 
Com base nos modelos ajustados para as cargas de combustíveis de até $25 \mathrm{~mm}$ e de combustível total, outros calibradores podem também ser construídos, embora não possam oferecer idêntica precisão, em razão dos menores índices de ajuste que esses modelos apresentaram.

\section{CONCLUSÕES}

Os resultados obtidos no trabalho permitiram concluir o seguinte:

1) A idade do plantio, o DAP, a altura dominante e a espessura da camada de acículas foram as variáveis que apresentaram melhores correlações com as cargas das diversas classes de material combustível das plantações de Pinus taeda;

2) A espessura da camada de acículas foi a variável independente que melhor se associou às diferentes classes de material combustível morto;

3) Os melhores ajustes obtidos foram referentes à carga de acículas.

4) As equações ajustadas para carga total de combustível perderam precisão em razão da variabilidade observada nas cargas de combustível verde e de diâmetro maior do que $2,5 \mathrm{~cm}$

5) Não se observou variação substancial da densidade de carga da camada de acículas em função da idade do povoamento, mantendo-se em torno de $0.025 \mathrm{~g} . \mathrm{cm}^{-3}$ a partir do quinto ano;

6) Os modelos que melhor representaram as cargas de combustíveis disponíveis ou perigosos foram aqueles ajustados para as acículas e para o material lenhoso morto com até $2,5 \mathrm{~cm}$ de diâmetro;

7) O melhor modelo para estimar a carga de acículas foi:

$$
W_{a}=2,356794 * S
$$

8) Estimativas razoáveis de material combustível total foram obtidas com o modelo

$$
W_{T}=3,35892 * S-1,87453 ;
$$

10) Os modelos lineares apresentaram maior $\mathrm{R}^{2}$ e menor erro padrão da estimativa do que os não lineares.

\section{REFERÊNCIAS}

AGEE, J. K. Fuel weights of under storygrown conifers in southern Oregon. Canada Journal of Forest Research, Ottawa, n. 13, p.648-656, 1983.

BATISTA, A. C. Determinação de umidade do material combustível sob povoamento de Pinus tadea L. Curitiba, 1984. Dissertação (Mestrado em Engenharia Florestal), Setor de Ciências Agrárias, Universidade Federal do Paraná.

BATISTA, A. C. Avaliação da queima controlada em povoamentos de Pinus taeda L. no Norte do Paraná. Curitiba, 1995. Tese (Doutorado em Engenharia Florestal), Setor de Ciências Agrárias, Universidade Federal do Paraná.

BRENDER, E. V. ; McNAB, W.H. ; WILLIANS S. Fuel accumulations in Piedmont loblolly pine plantations. North Carolina: U.S.D.A. Forest Service, 1976. (Research Note SE-233).

BROWN, J. K. Fuel and vegetation inventory procedures-white cap study, [ S.e : s.n.], 1971. (mimeografado).

BROWN, J. K. Handbook for inventorying downed woody material. Utah: U.S.D.A. Forest Service, 1974. (General Technical Report INT-16).

BROWN, J. K. ; BEVINS, C. D. Surface fuel loadings and predicted fire behavior for vegetation types in the northern Rocky Mountains. Utah: U.S.D.A. Forest Service, 1986. (Research Note INT-358).

BURROWS, N. D. et al. Prescribing low intensity fire to kill wildings in Pinus radiata plantations in Western Australia. Australian Forestry, Queen Victoria, v. 52, n. 1, p. 45-52, 1989.

CARLTON, D.W.; PICKFORD, S.G. Fuelbed changes with aging of slash from ponderosa pine thinnings. Journal of Forestry, Bethesda, v. 80, n. 2, p. 105-108, 1982. 
CHANDLER, C. et al. Fire in forestry: forest fire behavior and effects. New York : Wiley \& Sons, 1983.

DRAPER, N. R.; SMITH, A. Applied regression analysis. New York : Wiley \& Sons, 1966.

HINGSTON, F. J.; GALBRAITH, J. H. Nutrients in ash fall-out during forest fires in the southwest of Western Australia. Australian Forestry, Queen Victoria, v. 52, n. 4, p. 321326, 1989.

McCAW, W. L.; NEAL, J. E. ; SMITH, R. H. Fuel accumulation following prescribed burning in young evenaged stands de barri (Eucalyptus diversicolor). Australian Forestry, Queen Victoria, v. 59, n. 4, p. 171-177, 1996.

McRAE, D. J.; ALEXANDER, M. E. ; STOCKS, B. J. Measurement and description of fuels and fire behavior on prescribed burns: a handbook. Ontario : Canadian Forestry Service, 1979. p. 1-44. (Report 0-X-287).

PYNE, S. J. Wildland fire - Fire management in the United States. New York: Wiley \& Sons, 1984.

RIBEIRO, G. A. Estudos do comportamento do fogo e de alguns efeitos da queima controlada em povoamentos de Eucalyptus viminalis Labill em Três Barras, Santa Catarina. Curitiba, 1997. Tese (Doutorado em Engenharia Florestal) - Setor de Ciências Agrárias, Universidade Federal do Paraná.

SACKETT, S. S. Woody fuel particle size and specific gravity of southwestern tree species. Fort Collins : U.S.D.A. Forest Service, 1980. (Res. Note RM-389).

SCHIMMEL, J.; GRANSTRÖM, A. Fuel succession and fire behavior in the Swedish boreal forest. Canada Journal of Forest Research, Ottawa, n. 27, p. 1207-1216, 1997.
SCHMIDT, P. B. Determinação indireta da relação hipsométrica para povoa-mentos de Pinus taeda L. Curitiba, 1977. Dissertação (Mestrado em Engenharia Florestal) - Setor de Ciências Agrárias, Universidade Federal do Paraná.

SCHNEIDER, B. H.; BELL, D. T. A simple, effective technique for rapid measurement of fuels in low shrub communities. Australian Forest Research, East Melbourne, v. 15, n. 1, p. 79-84, 1985.

SOARES, R. V. Determinação da quantidade de material combustível acumulado em plantios de Pinus spp na região de Sacramento (MG). Revista Floresta, Curitiba, v. 10, n. 1, p. 48-62, 1979.

STEEL, R. G. D.; TORRIE, J. H. Principles and procedures of statistics - a Biometrical Approach. 2. Ed. New York: McGrow - Hill Book Co., 1980.

VANCLAY, J. K. Modelling forest growth and yield - applications to mixed tropical forests. Wallingford: CAB International, 1994.

WERKEMA, M. C. C.; AGUIAR, S. Análise de regressão : como entender o relacionamento entre as variáveis de um processo. Belo Horizonte: Fundação Christiano Ottoni, Escola de Engenharia da UFMG, 1996. (Série Ferramentas de qualidade, v. 7). 